\title{
Renal Nerve-Mediated Erythropoietin Release Confers Cardioprotection During Remote Ischemic Preconditioning
}

\author{
Toyoharu Oba, MD; Hideo Yasukawa, MD; Takanobu Nagata, MD; Sachiko Kyogoku, MD; \\ Tomoko Minami, MD; Michihide Nishihara, MD; Hideki Ohshima, MD; Kazutoshi Mawatari, MD; \\ Shoichiro Nohara, MD; Jinya Takahashi, MD; Yusuke Sugi, MD; Sachiyo Igata, PhD; \\ Yoshiko Iwamoto, MD; Hisashi Kai, MD; Hidehiro Matsuoka, MD; Makoto Takano, MD; \\ Hiroki Aoki, MD; Yoshihiro Fukumoto, MD; Tsutomu Imaizumi, MD
}

\begin{abstract}
Background: Remote ischemic preconditioning (RIPC) induced by transient limb ischemia is a powerful innate mechanism of cardioprotection against ischemia. Several described mechanisms explain how RIPC may act through neural pathways or humoral factors; however, the mechanistic pathway linking the remote organ to the heart has not yet been fully elucidated. This study aimed to investigate the mechanisms underlying the RIPC-induced production of Janus kinase (JAK)-signal transducer and activator of the transcription (STAT)-activating cytokines and cardioprotection by using mouse and human models of RIPC.

Methods and Results: Screened circulating cardioprotective JAK-STAT-activating cytokines in mice unexpectedly revealed increased serum erythropoietin (EPO) levels after RIP induced by transient ischemia. In mice, RIPC rapidly upregulated EPO mRNA and its main transcriptional factor, hypoxia-inducible factor-1a (HIF1a), in the kidney. Laser Doppler blood flowmetry revealed a prompt reduction of renal blood flow (RBF) after RIPC. RIPC activated cardioprotective signaling pathways and the anti-apoptotic Bcl-xL pathway in the heart, and reduced infarct size. In mice, these effects were abolished by administration of an EPO-neutralizing antibody. Renal nerve denervation also abolished RIPC-induced RBF reduction, EPO production, and cardioprotection. In humans, transient limb ischemia of the upper arm reduced RBF and increased serum EPO levels.
\end{abstract}

Conclusions: Based on the present data, we propose a novel RIPC mechanism in which inhibition of infarct size by RIPC is produced through the renal nerve-mediated reduction of RBF associated with activation of the HIF1a-EPO pathway. (Circ J 2015; 79: 1557-1567)

Key Words: Cardioprotection; Erythropoietin; JAK-STAT; Remote ischemic preconditioning; Renal nerve

$\mathbf{I}$ $\mathrm{n}$ acute myocardial infarction (MI), the infarct size determines the development of left ventricular remodeling, which is an important factor in the progression of cardiac insufficiency to overt heart failure, as well as an early marker of increased morbidity and mortality. ${ }^{1,2}$ Percutaneous coronary intervention and thrombolytic therapy can limit the infarct size and improve clinical outcome, but the survival after post-MI heart failure remains poor and has recently worsened. ${ }^{3}$ Thus, there is a need for new strategies to reduce infarct size in MI patients. It is important to clarify the underlying mechanisms of cardioprotective strategies against MI, which could aid in identifying novel therapeutic targets for reducing infarct size.

Murry et $\mathrm{al}^{4}$ discovered ischemic preconditioning, in which brief episodes of non-lethal ischemia followed by reperfusion reduce subsequent myocardial infarct size. Przklenk et al ${ }^{5}$ later reported that brief ischemia of the circumflex artery reduces subsequent infarct size in the territory of the left anterior descending artery, which was called remote preconditioning. Birnbaum et $\mathrm{al}^{6}$ extended this concept showing that transient ischemia in remote tissue of the limbs induces protection against subsequent myocardial ischemia. This inter-organ protection phenomenon is termed remote ischemic preconditioning (RIPC), and has powerful protective effects in the heart and in other vital organs. ${ }^{7,8}$

Animal and human studies have provided many lines of evidence suggesting that RIPC induced by transient limb ischemia

Received October 27, 2014; accepted February 23, 2015; released online March 31, 2015 Time for primary review: 15 days

Division of Cardiovascular Medicine, Department of Internal Medicine, Kurume University School of Medicine, Kurume (T.O., H.Y., T.N., S.K., T.M., M.N., H.O., K.M., S.N., J.T., Y.S., Y.I., H.K., H.M., Y.F.); Cardiovascular Research Institute, Kurume University, Kurume (H.Y., H.A., Y.F.); Division of Integrated Autonomic Function, Department of Physiology, Kurume University School of Medicine, Kurume (S.I., M.T.); and Fukuoka Sanno Hospital and International University of Health and Welfare, Fukuoka (T.I.), Japan

Mailing address: Hideo Yasukawa, MD, PhD, Division of Cardiovascular Medicine, Department of Internal Medicine, Kurume University School of Medicine, 67 Asahi-machi, Kurume 830-0011, Japan. E-mail: yahideo@med.kurume-u.ac.jp

ISSN-1346-9843 doi:10.1253/circj.CJ-14-1171

All rights are reserved to the Japanese Circulation Society. For permissions, please e-mail: cj@j-circ.or.jp 
confers cardioprotection during ischemia reperfusion injury or post-infarct left ventricular remodeling. ${ }^{7-9}$ Recent studies demonstrate improved prognosis in MI patients undergoing elective interventional or surgical coronary revascularization..$^{10,11}$ Regarding the mechanisms underlying RIPC, humoral factors and neural pathways, as well as their interaction, have been hypothesized to forward the protective signals to the heart. ${ }^{12-18}$ However, it has not yet been fully clarified what pathways link the remote organ to the target organ, including the heart.

Exogenous administration of erythropoietin (EPO) and granulocyte colony-stimulating factor (G-CSF) after MI reportedly induces cardiac protection by activating the cardioprotective Janus kinase (JAK)-signal transducer and activator of the transcription (STAT) pathway. ${ }^{19,20}$ Previously reported data indicate that the JAK-STAT pathway confers cardioprotection during MI by activating anti-apoptotic pathways and preserving mitochondrial functions. ${ }^{19-24}$ Mouse and human studies have demonstrated RIPC-induced activation of STAT3 and STAT5 in the heart, ${ }^{25,26}$ and Slagsvold et $\mathrm{al}^{27}$ reported that RIPC during coronary bypass surgery preserves myocardial mitochondrial function. Thus, RIPC apparently activates the JAK-STAT pathway and preserves mitochondrial function in the myocardium. We hypothesized that RIPC may reduce infarct size by producing JAK-STAT-activating cytokines. In the present study, we used mouse and human models of RIPC to investigate the mechanisms underlying the RIPC-induced production of JAKSTAT-activating cytokines and cardioprotection.

\section{Methods}

\section{Animals}

The studied mice were 8 - to 10 -week-old males in a Balb/c background.

\section{RIPC Induction}

In humans, RIPC was induced by inflating a 9-cm-wide blood pressure cuff positioned around the upper part of the arm. The cuff was inflated to $200 \mathrm{mmHg}$ for $5 \mathrm{~min}$ for ischemia, followed by a 5-min deflation, and this cycle was repeated 3 times..$^{10,11}$ In mice, RIPC was induced by $5 \mathrm{~min}$ of inflation of a pneumatic tourniquet positioned around the proximal right lower limb, followed by $5 \mathrm{~min}$ of reperfusion, and this cycle was repeated 4 times.9,15 The cessation of blood flow to the hindlimb was confirmed by a change in foot color and a reduced foot microcirculatory blood flow as measured by laser Doppler flowmetry.

\section{ELISA for EPO}

Mouse serum samples were analyzed for EPO by using an enzyme-linked immunosorbent assay (ELISA; R\&D Systems) according to the manufacturer's instructions. Circulating human EPO levels in serum were measured by radio-immunoassay (SRL, Tokyo, Japan).

\section{RNA Extraction and Real-Time Polymerase Chain Reaction (PCR)}

Total left ventricle RNA was isolated using TRIzol (Invitrogen), as described previously. ${ }^{28}$ Total RNA $(1 \mu \mathrm{g})$ was then reverse transcribed into cDNA. Real-time PCR assays were performed to assess the gene expressions of mouse EPO and GAPDH, using the corresponding primer pairs (Applied Biosystems; \#Mm01202755_m1, and \#Mm99999915_g1, respectively) and the StepOnePlus Real-Time PCR System (Applied Biosystems).

\section{Hypoxia Inducible Factor-1a (HIF1a) Immunohistochemical Staining}

Mouse kidneys were harvested $1 \mathrm{~h}$ after RIPC. Embedded sections were deparaffinized, and endogenous peroxidase activity was inhibited by treating the sections with $0.3 \% \mathrm{H}_{2} \mathrm{O}_{2}$ in PBS for $10 \mathrm{~min}$. After several washes with PBS, the sections were incubated for 20 min with blocking solution (Jackson ImmunoResearch) to block non-specific binding, followed by overnight incubation at $4{ }^{\circ} \mathrm{C}$ with the purified anti-hypoxia inducible factor- $1 \alpha$ (HIF1 $\alpha$ ) antibody (Abcam). Subsequently, the sections were incubated with an alkaline phosphatase-conjugated goat anti-rabbit IgG antibody for $30 \mathrm{~min}$. Signal amplification was achieved by incubating the slides for $30 \mathrm{~min}$ with Vectastain Elite Avidin-Biotin Complex solution (Vectastain ABC Kit, Vector), followed by incubation with Vectastain diaminobenzidine solution as the chromagen marker (Dako). ${ }^{28}$ For a negative staining control, goat serum was used in place of the HIF $1 \alpha$ antibody.

\section{Renal Blood Flow (RBF) Monitoring}

Mouse RBF was measured at 0 min and every 2 min during and after RIPC induction, using a laser Doppler blood flow imager (Laser Doppler Perfusion Imager System, moorLDI ${ }^{\mathrm{TM}}$ Mark 2, Moor Instruments). Before RBF scanning in the right kidney, mice were placed on a heating pad at $37^{\circ} \mathrm{C}$ to minimize temperature variations. In control mice, a sham procedure was performed, exposing the right kidney but maintaining right lower limb blood supply. The same technique was used to measure RBF after RIPC. The mean laser Doppler flux was analyzed at each time point, using computer processing software. Systemic systolic and diastolic blood pressure were measured in mice in the conscious state before and after RIPC using a tail-cuff method (TK-370C; UNI-COM, Tokyo, Japan). In humans, Doppler blood flow measurements of peak systolic velocity, end diastolic velocity, and aortic velocity were performed. Velocity waveforms were sampled in the right renal artery and the descending aorta.

\section{Model}

MI was produced by permanent ligation of the left coronary artery, as previously described. ${ }^{28}$ At $2 \mathrm{~h}$ after left coronary artery occlusion, the area at risk and initial infarct area were determined by perfusion with $5 \%$ Evans blue dye and subsequent incubation with triphenyltetrazolium chloride, as previously described. ${ }^{28}$

\section{Western Blot Analysis}

Western blot analysis was performed as described previously, ${ }^{28}$ using antibodies raised against phosphorylated STAT3, phosphorylated AKT, phosphorylated ERK1/2, Bcl-xL, Bax (New England BioLabs), HIF1 $\alpha$ (Abcam), and GAPDH. For HIF1 $\alpha$ western blotting, we prepared negative and positive controls of HIF $1 \alpha$ by rendering human astrocytes under normoxic and hypoxic conditions, respectively. Following previously reported methodology, ${ }^{29}$ hypoxia was induced by incubating the human astrocytes for $4 \mathrm{~h}$ in $1 \% \mathrm{O}_{2}$ and $5 \% \mathrm{CO}_{2}$ (balanced with $\mathrm{N}_{2}$ ) in a hypoxia chamber (ASTEC, Fukuoka, Japan).

\section{EPO Neutralization}

Neutralizing anti-mouse EPO antibody was purchased from R\&D Systems. The neutralizing effects of the antibody were verified by measuring circulating reticulocytes at 3 days after injection. Anti-EPO antibody (100 $\mu \mathrm{g} /$ mouse) was injected into mice through the tail vein at $24 \mathrm{~h}$ before RIPC or coronary occlusion. 

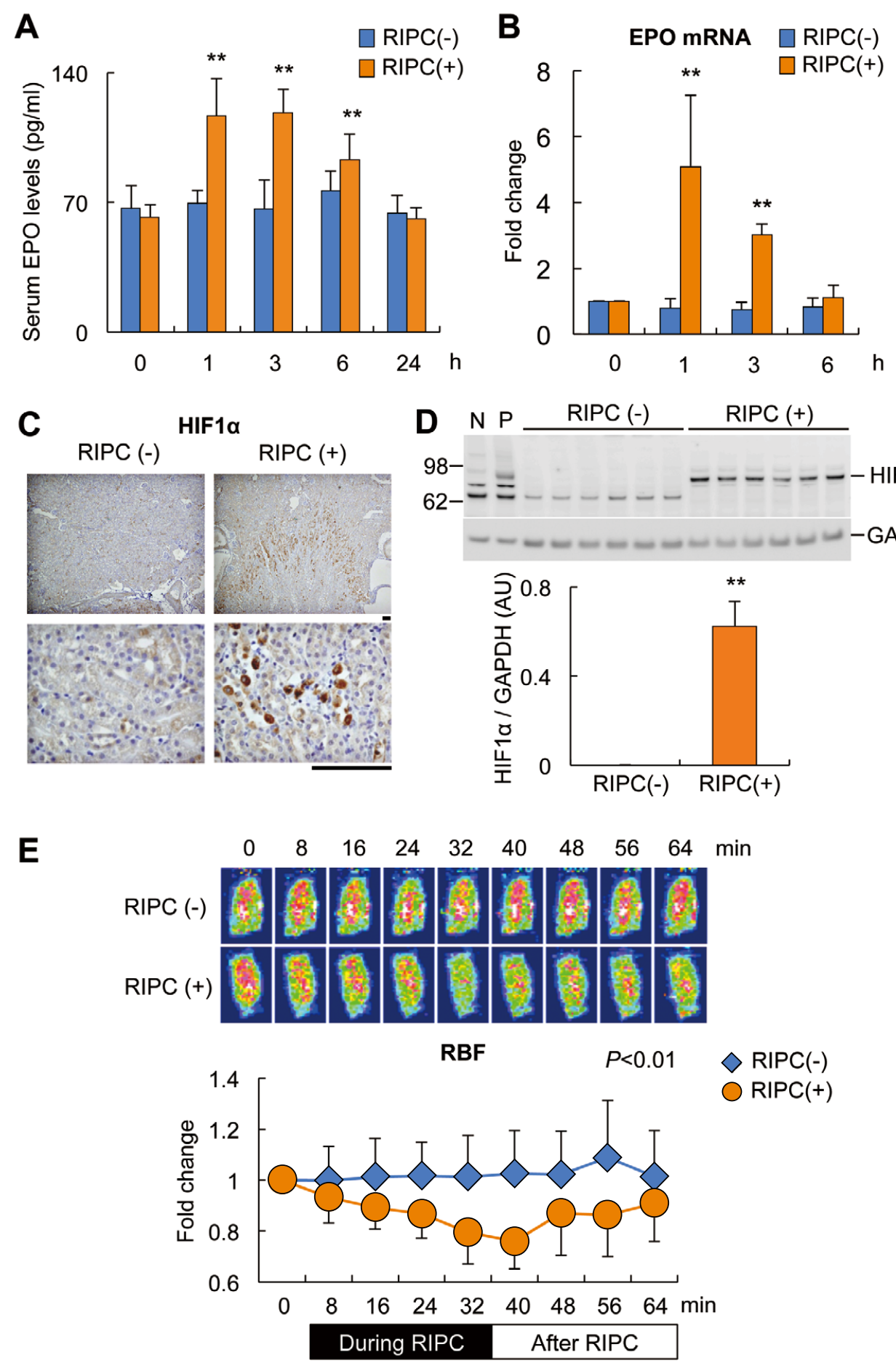

Figure 1. Activation of the renal hypoxia inducible factor $1 a$-erythropoietin (HIF1a-EPO) pathway after remote ischemic preconditioning (RIPC). (A) Serum levels of EPO in mice after RIPC, determined by an enzyme-linked immunosorbent assay (ELISA) at the indicated time ( $n=5$ per group). ${ }^{*} P<0.01$ vs. pre-RIPC. (B) EPO expression time-course in the kidney after RIPC, analyzed by real-time polymerase chain reaction (PCR) ( $n=5$ per group). Values are normalized to GAPDH, and expressed as a fold change from pre-RIPC values. ${ }^{* *} \mathrm{P}<0.01$ vs. pre-RIPC. (C) HIF1a expression in the kidney at $1 \mathrm{~h}$ after RIPC. Paraffin sections with and without RIPC were stained with anti-HIF1a antibody (brown). Scale bar=100 $\mu \mathrm{m}$. (D) HIF1a expression in the kidney at $1 \mathrm{~h}$ after RIPC was examined by western blot analysis with the HIF1a antibody. Negative (N) and positive (P) controls of HIF1a were prepared from human astrocytes cultured under conditions of normoxia and hypoxia, respectively. ${ }^{\star \star} \mathrm{P}<0.01 \mathrm{vs}$. mice without RIPC. (E) Laser Doppler image of mouse renal blood flow (RBF) at the indicated times during and after RIPC ( $n=7$ per group); pooled data are shown, with values expressed as fold-change from pre-RIPC values. 

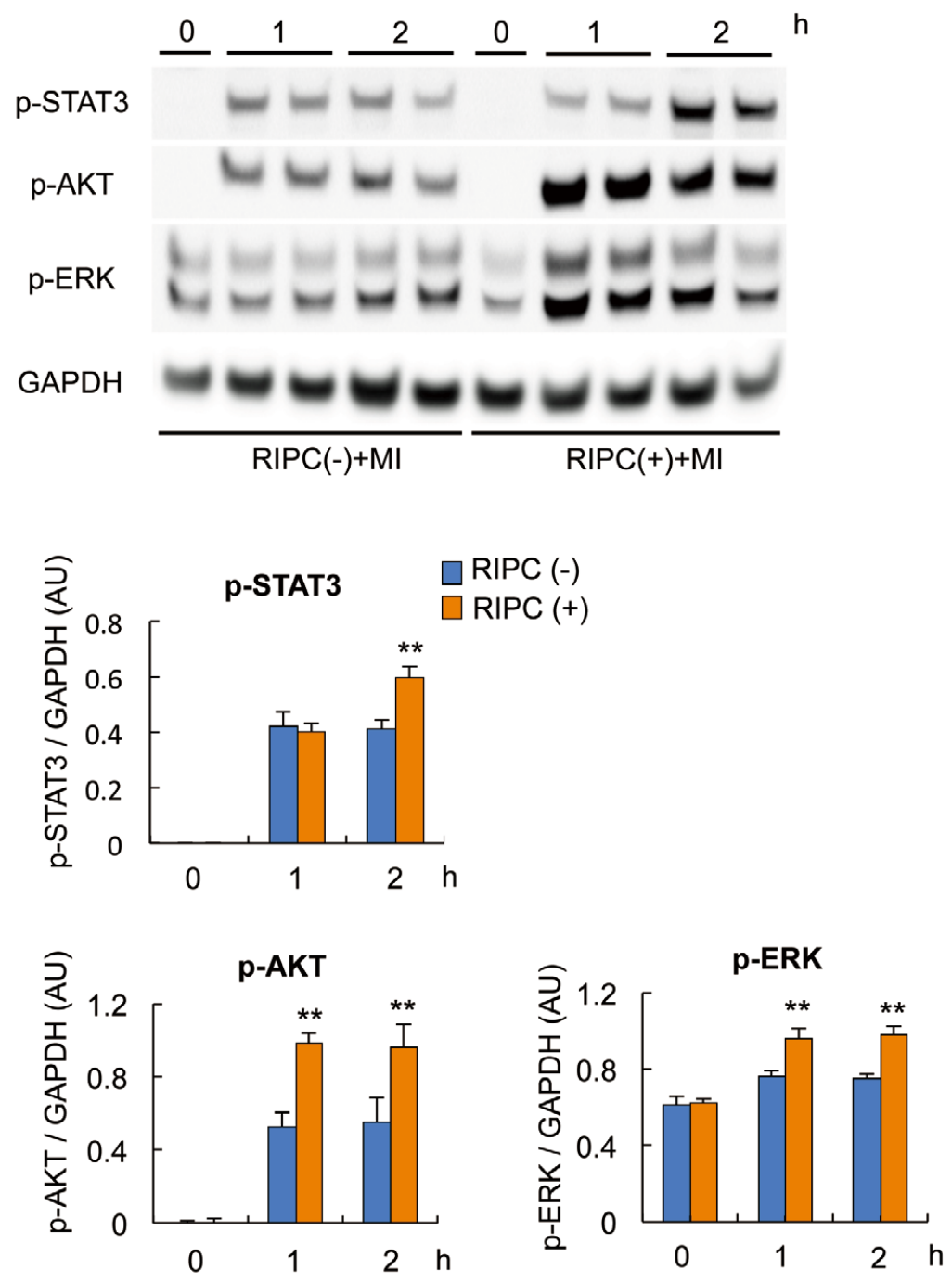

Figure 2. Enhanced activation of cardioprotective signaling pathways by remote ischemic preconditioning (RIPC) in infarct hearts. Total cell lysates were prepared from hearts of mice with or without RIPC at the indicated times after myocardial infarction (MI). Lysates were blotted with antibodies raised against phosphorylated STAT3 (p-STAT3), phosphorylated AKT (p-AKT), phosphorylated ERK1/2 (p-ERK1/2), and GAPDH. Bar graphs represent quantitative differences in p-STAT3, p-AKT, and p-ERK1/2 expression ( $n=5$ per group). ${ }^{* *} P<0.01$ vs. mice without RIPC. AU, arbitrary units.

\section{Renal Denervation and Sham Surgery}

Mice were anesthetized with isoflurane. Renal denervation was performed by stripping the nervous and connective tissues that passed to and along the course of the renal artery and veins of both kidneys. These vessels were then painted with a solution of $10 \%$ phenol in ethanol through a midline abdominal incision. ${ }^{30}$ Sham-operated control mice received only a midline incision, and the renal nerves were left intact. At the end of the experiment, renal denervation was verified by analyzing the renal norepinephrine content in the kidney. ${ }^{30}$

\section{Study Approval}

Enzyme-linked immunosorbent assay using serum from healthy volunteers and a color Doppler ultrasound examination of healthy volunteers, were declared exempt from requiring approval by the institutional review board of Kurume University. Written informed consent was obtained from each healthy volunteer. All experimental procedures with mice were performed according to guidelines for experiments in animals established by the Kurume University Animal Care and Treatment Committee.

\section{Statistical Analysis}

Data are expressed as mean \pm standard error. Multiple group comparisons were performed using one-way analysis of variance (ANOVA). Comparisons between 2 groups were analyzed using a 2-tailed Student's t-test or a 2-way ANOVA. A P value of less than 0.05 was considered to indicate statistical significance.

\section{Results}

\section{Activation of the Renal HIF1a-EPO Pathway After RIPC}

Following RIPC induction in mice, we measured the serum levels of several JAK-STAT-activating cytokines. RIPC did not alter serum levels of G-CSF or leukemia inhibitory factor (data not shown), but it unexpectedly increased serum EPO levels (Figure 1A). As EPO is mainly produced in the kidney, we next used real-time PCR to examine EPO mRNA expression in the kidney after RIPC, and found that it rapidly increased (Figure 1B). The main transcriptional factor involved in EPO expression is HIF $1 \alpha$. Thus, we performed immunohistochemical staining of HIF1 $\alpha$ in the kidney, which revealed low HIF1 $\alpha$ expression without RIPC. In contrast, immediately after RIPC, 


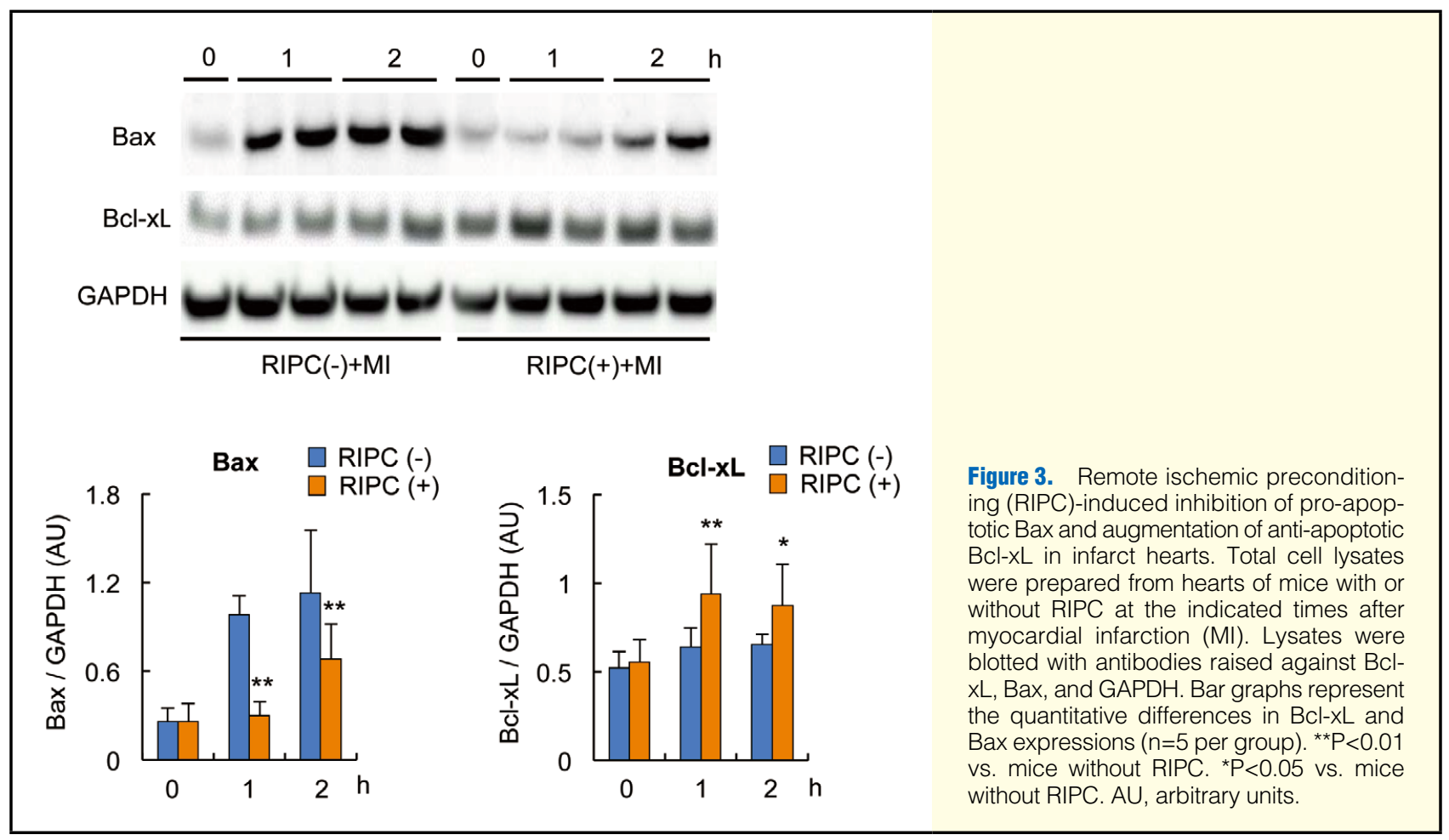

we observed markedly higher HIF $1 \alpha$ expression in the border area between the medulla and the cortex of the kidney (Figure 1C). We also performed western blot analysis to examine the induction of HIF $1 \alpha$ expression in the kidney after RIPC. HIF $1 \alpha$ expression was absent from the kidney without RIPC, but was detected in the kidney at $1 \mathrm{~h}$ after RIPC (Figure 1D). The detected band from the kidney after RIPC was of a similar molecular weight compared with that observed in the positive control of HIF $1 \alpha$ from astrocytes cultured under hypoxia (Figure 1D). These results indicate that RIPC rapidly activated the HIF1 $\alpha$-EPO pathway in the kidney.

\section{Rapid Reduction of RBF After RIPC}

Circulating EPO levels are regulated by renal oxygen supply, which is determined by hemoglobin concentration, hemoglobin oxygen saturation, and RBF. ${ }^{31}$ RIPC did not alter hemoglobin concentration or hemoglobin oxygen saturation (data not shown), but laser Doppler blood flowmetry revealed a rapid and significant reduction of RBF during and after RIPC (Figure 1E). Systemic systolic and diastolic blood pressure values measured by a tail-cuff method in mice after RIPC were comparable to values obtained in mice without RIPC (Figure S1). This suggested that RIPC promptly reduced RBF level, which activated the renal HIF1 $\alpha$-EPO pathway, and subsequently increased serum EPO levels.

\section{Enhanced Activation of Cardioprotective Signaling Pathways by RIPC in Infarct Hearts}

Next, we investigated whether RIPC initiated EPO-activated cardioprotective signaling. In infarct hearts, RIPC led to greater activation of cardioprotective signaling, STAT3, AKT, and extracellular-signal-regulated kinases 1 and 2 (ERK1/2), compared to that seen without RIPC (Figure 2). RIPC also led to lower pro-apoptotic Bax expression and greater anti-apoptotic $\mathrm{Bcl}-\mathrm{xL}$ expression, compared to without RIPC (Figure 3). These results supported the concept that RIPC initiated EPO-

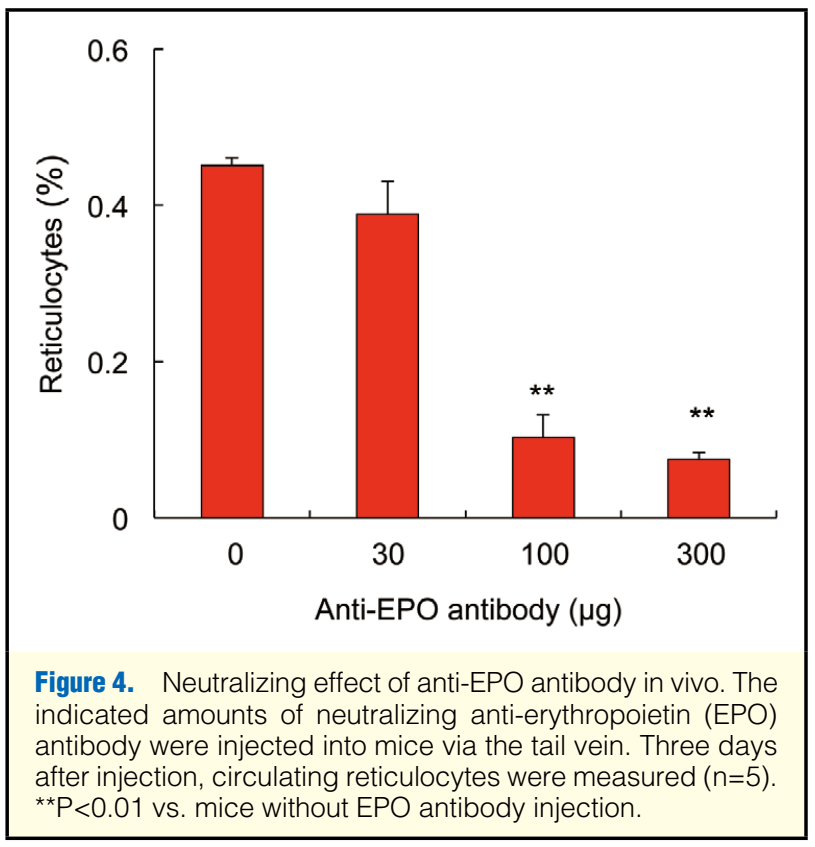

activating cardioprotective signaling pathways, as well as inhibited cardiac apoptotic signals in mice after MI.

\section{Abolishment of RIPC-Induced Cardioprotection by Anti-EPO Neutralizing Antibody}

To further validate the role of RIPC-induced EPO expression in the activation of cardioprotective signaling pathways and the reduction of infarct size, we administered an anti-EPO neutralizing antibody to mice after MI. Three days after anti-EPO antibody injection, we observed a marked reduction in circu- 

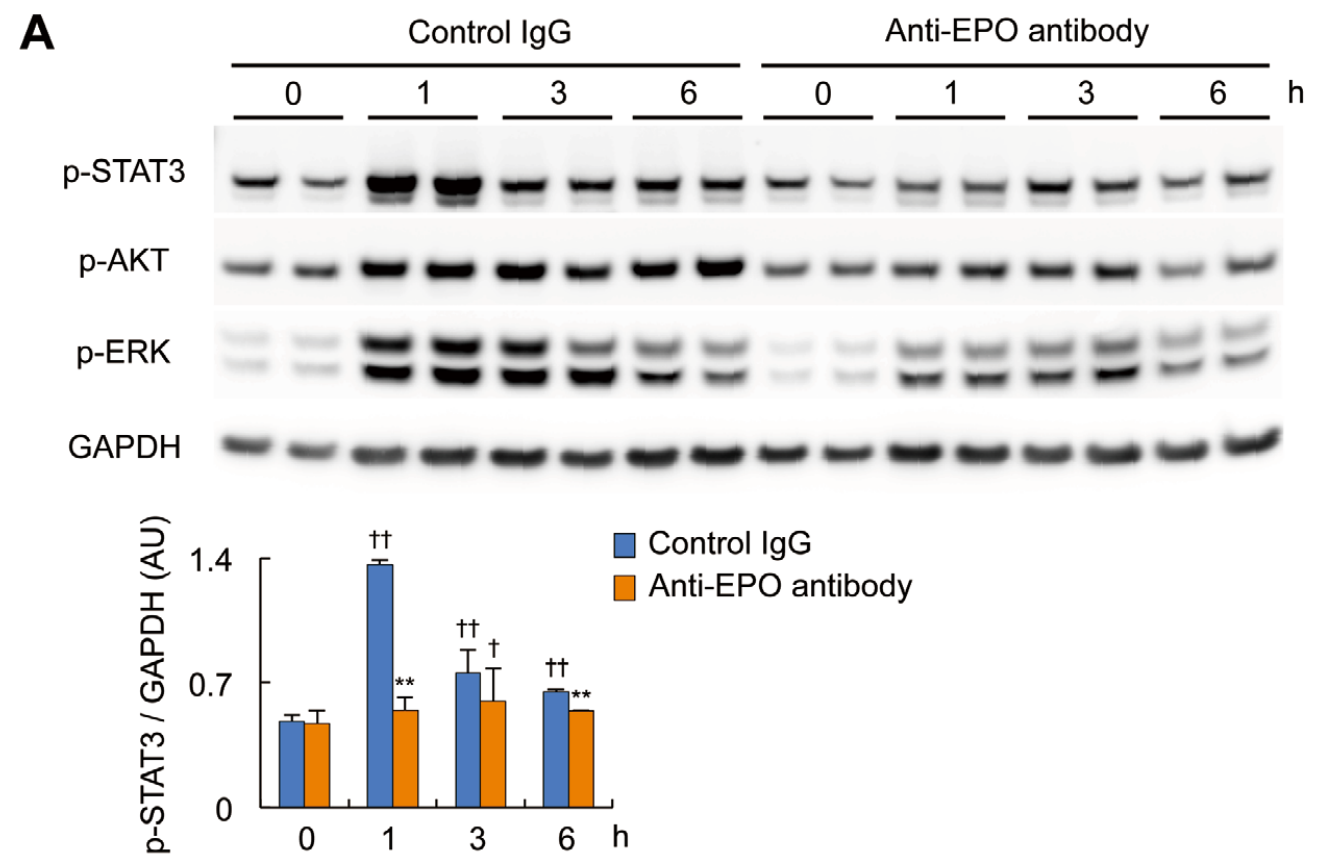

Control lgG

Anti-EPO antibody
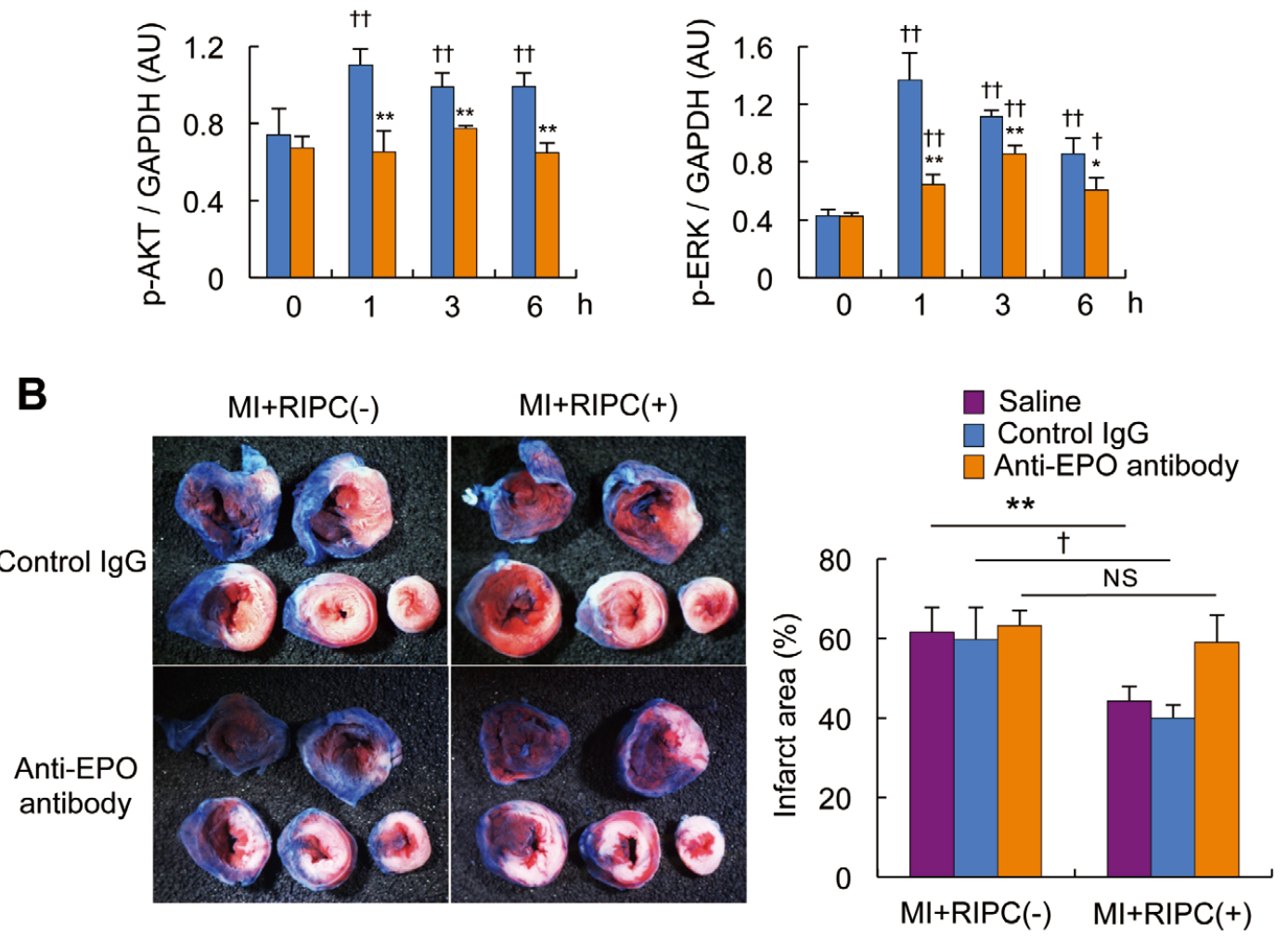

Figure 5. Cardioprotection abolishment by anti-EPO neutralizing antibody. (A) Total cell lysates were prepared from the hearts of mice injected with control immunoglobulin (Ig)G or anti-erythropoietin (EPO) antibody at the indicated times after remote ischemic preconditioning (RIPC). Lysates were blotted with antibodies raised against phosphorylated STAT3 (p-STAT3), phosphorylated AKT (p-AKT), phosphorylated ERK1/2 (p-ERK1/2), and GAPDH. Bar graphs represent quantitative differences in p-STAT3, $p-A K T$, and $p$-ERK $1 / 2$ expressions ( $n=5$ per group). $A U$, arbitrary units. ${ }^{\dagger} P<0.01$ vs. pre-RIPC; ${ }^{\dagger} P<0.05$ vs. pre-RIPC; ${ }^{* \star} P<0.01$ vs. control IgG-treated group; ${ }^{\star} \mathrm{P}<0.05$ vs. control IgG-treated group. (B) Evans blue dye and triphenyltetrazolium chloride (TTC) staining of mouse hearts at $2 \mathrm{~h}$ after myocardial infarction (MI) ( $\mathrm{n}=5$ per group). Mice were injected with saline, control lgG, or anti-EPO neutralization antibody; then treated with or without RIPC; followed by MI induction. Infarct size was expressed as a percentage of the area at risk (AAR) in each group; pooled data are shown. ${ }^{*} \mathrm{P}<0.01 \mathrm{vs}$. saline without RIPC; ${ }^{\mathrm{P}} \mathrm{P}<0.01 \mathrm{vs}$. control IgG without RIPC. NS, not significant. 
lating reticulocytes, and confirmed that $100 \mu \mathrm{g}$ of the anti-EPO antibody was sufficient to neutralize EPO (Figure 4). In the hearts of mice injected with control IgG, RIPC significantly increased the phosphorylation of STAT3, AKT, and ERK1/2. In contrast, injection of an anti-EPO neutralizing antibody abolished the RIPC-induced activation of STAT3, AKT, and ERK1/2 (Figure 5A). Correspondingly, RIPC significantly reduced infarct size in mice with control IgG injection, while injection of an anti-EPO neutralizing antibody cancelled out the RIPCinduced attenuation of infarct size (Figure 5B).

\section{Renal Nerve Denervation Abolished Effects of RIPC}

RBF was promptly reduced with RIPC, and we hypothesized that the efferent renal nerve might mediate this reduction. Thus, we performed bilateral renal denervation in mice to examine the role of the renal nerve system with respect to the effects of RIPC. We observed markedly reduced norepinephrine content in the kidney, confirming complete denervation (Figure 6). Systemic systolic and diastolic blood pressure at baseline in renal denervated mice were comparable to values obtained in mice without renal denervation, and these values were not altered after RIPC (Figure S1). The RIPC-induced reduction of $\mathrm{RBF}$ and increase in serum EPO production were abolished in the renal denervated mice (Figures 7A,B). At baseline, the absolute value blood perfusion units (BPU) of RBF were comparable between mice with and without renal denervation (Figure S2). After PIPC in mice with and without renal denervation, the alteration patterns of RBF shown by BPU were comparable to those shown by a fold change (Figures S2,7A). We observed that renal denervation also abolished the RIPCinduced amelioration of initial infarct size (Figure 7C). These results indicated that the effects of RIPC on EPO secretion and cardioprotection are mediated through the efferent renal nerve.

\section{Increased Serum EPO Levels and Reduced RBF After RIPC in Humans}

Finally, we evaluated whether transient ischemia of the upper arm would increase serum EPO levels and reduce RBF in healthy human volunteers. Consistent with the mouse data, RIPC induction in humans significantly increased serum EPO levels (Figure 8A). Other cardioprotective JAK-STAT-activating cytokines, such as interleukin-6 and G-CSF, were not altered after RIPC (Figure S3). A Color Doppler ultrasound revealed that the peak systolic and end diastolic renal-to-aortic flow velocity ratios were significantly reduced after RIPC in healthy volunteers (Figure 8B), indicating that RBF was reduced after RIPC. These results suggest that RIPC may activate the renal nerve-EPO pathway in humans.

\section{Discussion}

In the present study, we have investigated the cardioprotective mechanism active during RIPC in mice and humans. In mice, RIPC induced activation of cardioprotective signaling pathways and reduction of initial infarct size, both of which were abolished by administration of an EPO-neutralizing antibody, suggesting central involvement of EPO in RIPC-induced cardioprotection in MI. Renal nerve denervation of mice also abolished RIPC-induced RBF reduction, EPO production, and cardioprotection. In humans, transient limb ischemia of the upper arm reduced RBF and increased serum EPO levels. These findings suggest that renal efferent nerve-mediated activation of the HIF1 $\alpha$-EPO pathway underlies RIPC-induced cardioprotection.

Efferent renal sympathetic nerve activity dose-dependently

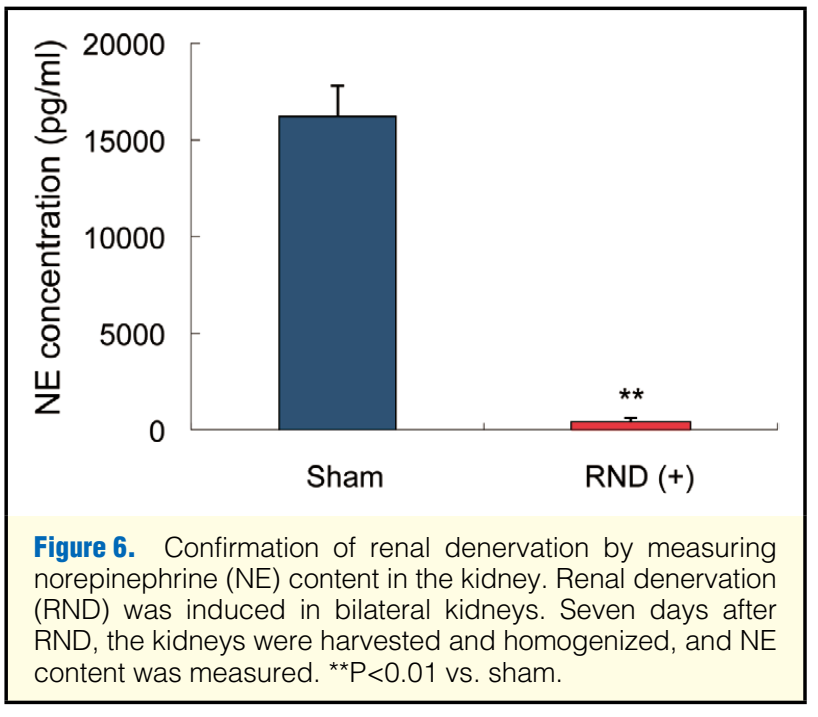

affects RBF, with high-frequency renal nerve stimulation decreasing $\mathrm{RBF}$ and increasing renal vascular resistance in rats. ${ }^{32}$ However, the role of the renal nerve in regulation of EPO production is controversial. Eckardt et $\mathrm{al}^{33}$ report that renal innervation plays a dispensable role in controlling EPO production under both normal and hypoxic conditions. On the other hand, renal denervation and pharmacological sympathetic nerve blockade abolish hypoxia-induced renal EPO production, ${ }^{34-36}$ suggesting an important role of the renal nerve. In the present study, the RBF reduction and EPO production induced by repeated limb tissue hypoxia were abolished by renal denervation, representing the first demonstration that RIPC reduces $\mathrm{RBF}$ and induces EPO production, likely through renal sympathetic nerve activation. The discrepancies regarding the renal nerve's role in EPO production may relate to differences in tissue hypoxia duration and intensity.

Exogenous administration of EPO reportedly leads to significant attenuation of $\mathrm{MI},{ }^{37}$ and increased endogenous serum EPO is associated with beneficial effects on MI pathophysiology. ${ }^{38-41}$ Namiuchi et $\mathrm{al}^{38}$ reported that MI patients with higher serum EPO (median, $39.5 \mathrm{mU} / \mathrm{ml}$ ) showed smaller infarct size than those with low serum EPO (median, $14.7 \mathrm{mU} / \mathrm{ml}$ ). Nakamura et $\mathrm{al}^{40}$ report that the time-dependent increase of endogenous serum EPO (median rising from 19.8 to $39.1 \mathrm{mU} / \mathrm{ml}$ ) is associated with improvement of cardiac function in MI patients. In the present study, the median serum EPO levels in healthy volunteers significantly increased from 19.6 to $32.2 \mathrm{mU} / \mathrm{ml}$ after RIPC. The median post-RIPC level was close to previously reported higher serum EPO levels in MI patients, suggesting potential biological significance of increased serum EPO levels after RIPC in humans. However, the biological effect of RIPC-induced EPO increase remains to be determined; for example, by measuring red blood cell or reticulocyte numbers after RIPC.

Infarct sizes in renal denervated mice were smaller than those in mice without renal denervation (Figures 5B,7C). Several previous studies have demonstrated that renal denervation attenuates left ventricular remodeling after MI, ${ }^{42-44}$ suggesting that renal denervation has cardioprotective effects on MI development. Our present study results indicated that RIPC did not induce additional preventive effects on infarct size in mice with renal denervation, suggesting that the renal nerve plays an important role in cardioprotection during RIPC. 
A

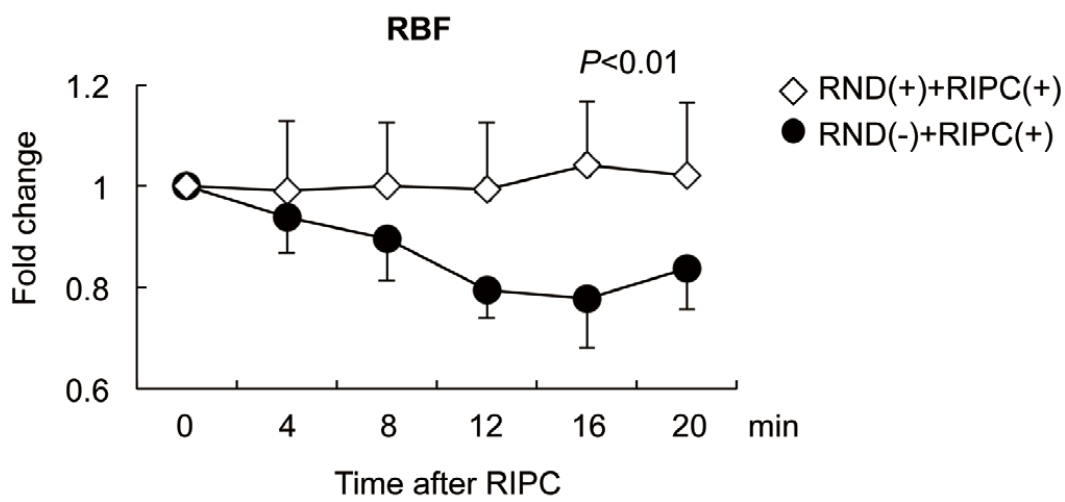

B

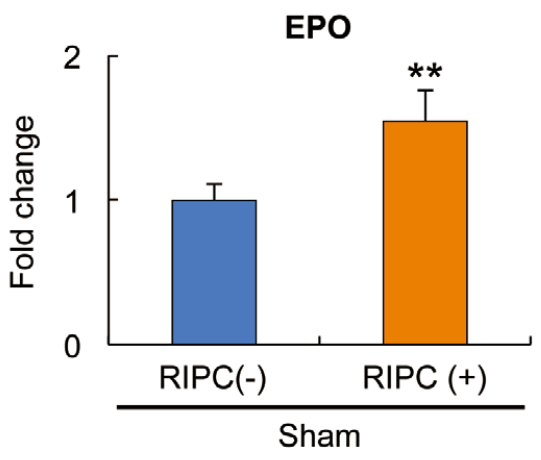

C

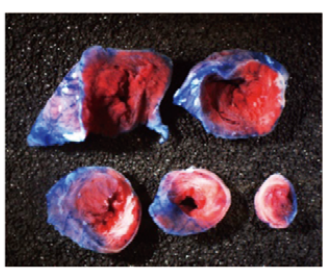

MI+RIPC(-)

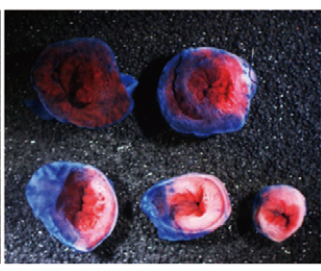

$\mathrm{Ml}+\mathrm{RIPC}(+)$
RND (+)
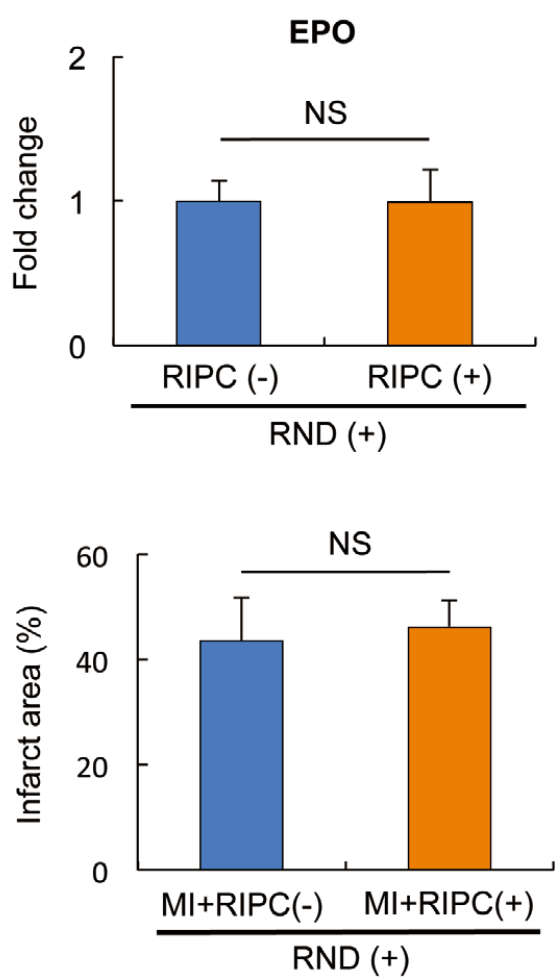

Figure 7. Renal denervation abolished effects of RIPC. (A) Laser Doppler image of renal blood flow (RBF) in mice with or without renal denervation (RND) at the indicated time after remote ischemic preconditioning (RIPC) ( $n=7$ per group). RBF was measured 7 days after RND induction. Pooled RBF data are shown, with values expressed as fold-change from the pre-RIPC values. (B) The effects of RIPC on serum erythropoietin (EPO) levels were determined in mice with RND or sham operation ( $n=5$ per group). Values are expressed as fold-change from the values of mice without RIPC. ${ }^{* *} \mathrm{P}<0.01$ vs. mice without RIPC; NS, not significant. (C) The effects of RIPC on initial infarct size were determined by staining with Evans blue dye and triphenyltetrazolium chloride (TTC) in renal denervated mice ( $n=5$ per group). Myocardial infarction (MI) was induced 7 days after RND induction. Infarct size was expressed as a percentage of the area at risk (AAR) in each group. NS, not significant.

Our present study did not investigate how repeated limb ischemia activated muscle afferent nerves. Previous reports have demonstrated the abolishment of RIPC-induced cardioprotective effects by pre-treatment with the ganglionic blocker, hexamethonium, or by prior resection of the femoral nerve..$^{15,45-47}$ It has been suggested that autocoids, such as adenosine or bradykinin, may activate a local afferent neural pathway within the remote organ or tissue, causing efferent renal nerve activation via the brain. ${ }^{45-47}$ In such a case, the afferent nerve activation of the lower limb would be important in the mechanism of RIPC-induced cardioprotection.

Our present findings support observations of RIPC-induced cardioprotection, as well as provide novel conceptual information, particularly regarding the importance of the kidney in this process. Previous reports have demonstrated the interorgan protective mechanism between 2 organs (eg, the limb and heart) during RIPC, and here we demonstrate the additional importance of the kidney. Chronic kidney disease has been associated with increased mortality in patients with MI. The present study results indicate that the kidney may be 
A

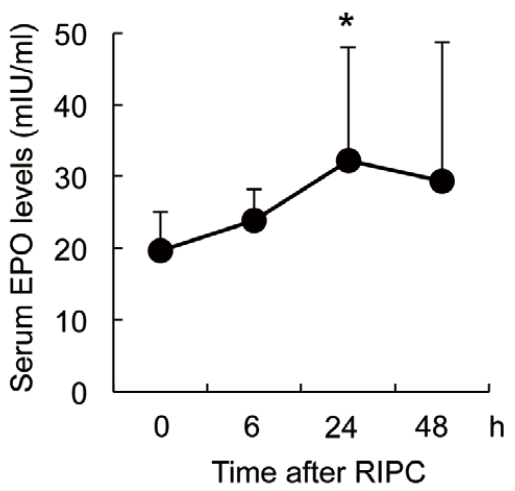

B

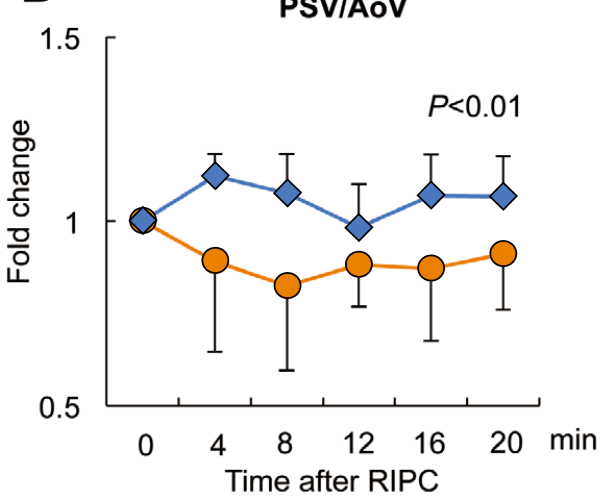

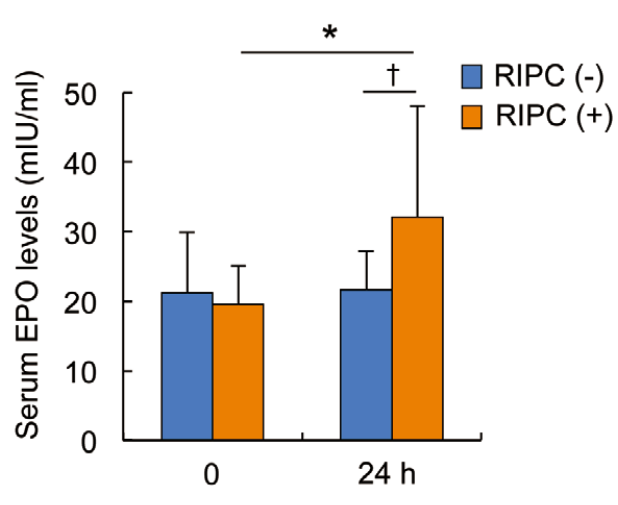

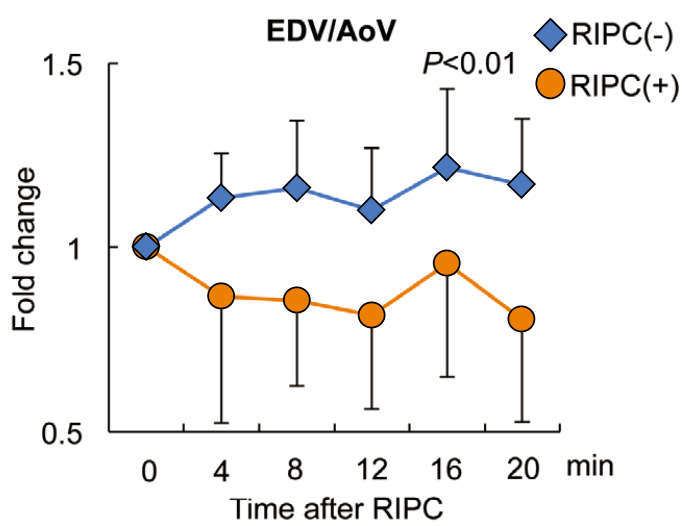

C

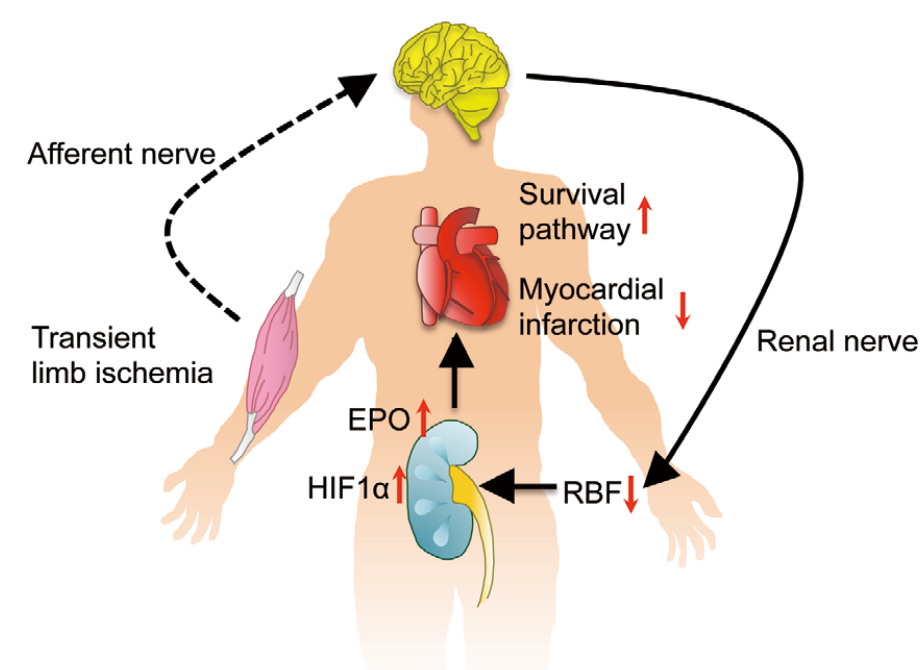

Figure 8. Increased serum erythropoietin (EPO) levels and reduced renal blood flow (RBF) after remote ischemic preconditioning (RIPC) in humans. (A) Serum EPO level time-course after RIPC in humans was determined by ELISA (Left panel, $n=10)$. Serum EPO levels before and $24 \mathrm{~h}$ after RIPC (Right panel, $n=10$ per group). ${ }^{\star} P<0.05$ vs. pre-RIPC; ${ }^{\top} P<0.05$ vs. after $24 \mathrm{~h}$ without $R I P C$. (B) Color Doppler ultrasound measurements of RBF in humans ( $n=8$ per group). The graph shows the ratio of renal artery peak systolic velocity (PSV) to descending aorta systolic velocity (AoV) (Left panel), and the ratio of renal artery end-diastolic velocity (EDV) to AoV (Right panel). Values are expressed as fold-change from the pre-RIPC values. (C) Working model for activation of the renal nerve-mediated hypoxia inducible factor 1a-erythropoietin (HIF1a-EPO) pathway in RIPC-induced cardioprotection. Repeated transient limb ischemia induces reduced RBF through the renal nerve pathway and, in turn, reduced RBF may decrease the oxygen supply that activates the HIF1a-EPO pathway. RIPC-induced EPO activates cardiac survival pathways, and inhibits apoptotic signals, resulting in the prevention of ischemic myocardial injury. 
centrally involved in the defense mechanism against ischemic organ injury through activation of the renal nerve-mediated HIF $1 \alpha$-EPO pathway. EPO reportedly has cytoprotective effects on vital organs, including the heart, brain, spinal cord, liver, and kidney. ${ }^{20,41}$ Therefore, the activation of this machinery during RIPC may play a role in protecting the heart - as well as other vital organs, such as the brain - as an intrinsic defense mechanism against ischemia-induced tissue injury.

\section{Study Limitations}

The present study has several limitations. To clarify the mechanism underlying the RIPC-induced reduction of RBF, we measured the renal norepinephrine content immediately after RIPC, and found it to be comparable between mice with and without RIPC (Figure S4). Although it has been shown that measuring renal norepinephrine content is the standard method to verify renal nerve denervation, ${ }^{30}$ tissue content of norepinephrine may not directly reflect dynamic sympathetic nerve activity comparing with other established methods, such as norepinephrine spillover ratio or electrode-based measurement of sympathetic nerve activity. Thus, it remains necessary to further investigate renal sympathetic nerve activity using the established methods.

\section{Conclusions}

Based on our present findings, we propose a novel RIPC mechanism, illustrated in Figure 8C. Briefly, transient limb ischemia activates the afferent muscle nerves and the renal efferent nerves through the central nervous system, reduces RBF, induces renal HIF $1 \alpha$ expression, and produces and releases EPO from the kidney. In turn, the released EPO activates cardioprotective signals and reduces initial infarct size. This is the first report to potentially clarify how a neural pathway through renal-nerve mediates the signal from a preconditioned remote organ to the target organ. The activation of the renal nerve-mediated HIF $1 \alpha$ EPO pathway during RIPC may play a role in protecting the heart as an intrinsic defense mechanism against myocardial injury after ischemia.

\section{Acknowledgments}

We thank Kimiko Kimura, Miyuki Nishigata, Makiko Kiyohiro, Katsue Shiramizu, and Miho Nakao for their excellent technical assistance. This study was supported, in part, by a grant for the Science Frontier Research Promotion Centers (Cardiovascular Research Institute); by Grants-in-Aid for Scientific Research (T.O., H.Y., T.I.) from the Ministry of Education, Science, Sports, and Culture, Japan; by a Research Grant for Cardiovascular Diseases from the Kimura Memorial Heart Foundation (H.Y.); and by a Japanese Ministry of Health, Labour and Welfare Research Grant (T.I.).

\section{Disclosures}

No conflicts of interest to be declared.

\section{References}

1. Mann DL, Bristow MR. Mechanisms and models in heart failure: The biomechanical model and beyond. Circulation 2005; 111: $2837-$ 2849.

2. Chareonthaitawee P, Christian TF, Hirose K, Gibbons RJ, Rumberger JA. Relation of initial infarct size to extent of left ventricular remodeling in the year after acute myocardial infarction. J Am Coll Cardiol 1995; 25: 567-573.

3. Chen J, Hsieh AF, Dharmarajan K, Masoudi FA, Krumholz HM. National trends in heart failure hospitalization after acute myocardial infarction for Medicare beneficiaries: 1998-2010. Circulation 2013; 128: $2577-2584$.

4. Murry CE, Jennings RB, Reimer KA. Preconditioning with ischemia: A delay of lethal cell injury in ischemic myocardium. Circulation 1986; 74: 1124-1136.
5. Przyklenk K, Bauer B, Ovize M, Kloner RA, Whittaker P. Regional ischemic 'preconditioning' protects remote virgin myocardium from subsequent sustained coronary occlusion. Circulation 1993; 87: $893-$ 899.

6. Birnbaum Y, Hale SL, Kloner RA. Ischemic preconditioning at a distance: Reduction of myocardial infarct size by partial reduction of blood supply combined with rapid stimulation of the gastrocnemius muscle in the rabbit. Circulation 1997; 96: 1641-1646.

7. Kloner RA. Clinical application of remote ischemic preconditioning. Circulation 2009; 119: 776-778.

8. Hausenloy DJ, Yellon DM. The therapeutic potential of ischemic conditioning: An update. Nat Rev Cardiol 2011; 8: 619-629.

9. Wei M, Xin P, Li S, Tao J, Li Y, Li J, et al. Repeated remote ischemic postconditioning protects against adverse left ventricular remodeling and improves survival in a rat model of myocardial infarction. Circ Res 2011; 108: 1220-1225.

10. Thielmann M, Kottenberg E, Kleinbongard P, Wendt D, Gedik N, Pasa S, et al. Cardioprotective and prognostic effects of remote ischaemic preconditioning in patients undergoing coronary artery bypass surgery: A single-centre randomised, double-blind, controlled trial. Lancet 2013; 382: 597-604.

11. Davies WR, Brown AJ, Watson W, McCormick LM, West NE, Dutka DP, et al. Remote ischemic preconditioning improves outcome at 6 years after elective percutaneous coronary intervention: The CRISP stent trial long-term follow-up. Circ Cardiovasc Interv 2013; 6: $246-251$

12. Hausenloy DJ, Yellon DM. Remote ischaemic preconditioning: Underlying mechanisms and clinical application. Cardiovasc Res 2008; 79: $377-386$.

13. Shimizu M, Tropak M, Diaz RJ, Suto F, Surendra H, Kuzmin E, et al. Transient limb ischaemia remotely preconditions through a humoral mechanism acting directly on the myocardium: Evidence suggesting cross-species protection. Clin Sci (Lond) 2009; 117: 191-200.

14. Dickson EW, Lorbar M, Porcaro WA, Fenton RA, Reinhardt CP, Gysembergh A, et al. Rabbit heart can be "preconditioned" via transfer of coronary effluent. Am J Physiol 1999; 277: H2451-H2457.

15. Lim SY, Yellon DM, Hausenloy DJ. The neural and humoral pathways in remote limb ischemic preconditioning. Basic Res Cardiol 2010; 105: 651-655.

16. Redington KL, Disenhouse T, Strantzas SC, Gladstone R, Wei C, Tropak MB, et al. Remote cardioprotection by direct peripheral nerve stimulation and topical capsaicin is mediated by circulating humoral factors. Basic Res Cardiol 2012; 107: 241.

17. Seino Y. Remote ischemic conditioning: From organs/tissues to organs. Circ J 2013; 77: 2883-2885.

18. Igarashi $\mathrm{G}$, Iino $\mathrm{K}$, Watanabe $\mathrm{H}$, Ito $\mathrm{H}$. Remote ischemic pre-conditioning alleviates contrast-induced acute kidney injury in patients with moderate chronic kidney disease. Circ J 2013; 77: 3037-3044.

19. Harada M, Qin $Y$, Takano H, Minamino T, Zou Y, Toko H, et al. G-CSF prevents cardiac remodeling after myocardial infarction by activating the Jak-Stat pathway in cardiomyocytes. Nat Med 2005; 11: $305-311$.

20. Riksen NP, Hausenloy DJ, Yellon DM. Erythropoietin: Ready for prime-time cardioprotection. Trends Pharmacol Sci 2008; 29: 258267.

21. Hirata A, Minamino T, Asanuma H, Fujita M, Wakeno M, Myoishi $\mathrm{M}$, et al. Erythropoietin enhances neovascularization of ischemic myocardium and improves left ventricular dysfunction after myocardial infarction in dogs. J Am Coll Cardiol 2006; 48: 176-184.

22. Obana M, Maeda M, Takeda K, Hayama A, Mohri T, Yamashita T, et al. Therapeutic activation of signal transducer and activator of transcription 3 by interleukin-11 ameliorates cardiac fibrosis after myocardial infarction. Circulation 2010; 121: 684-691.

23. Bolli R, Dawn B, Xuan YT. Role of the JAK-STAT pathway in protection against myocardial ischemia/reperfusion injury. Trends Cardiovasc Med 2003; 13: $72-79$.

24. Barry SP, Townsend PA, Latchman DS, Stephanou A. Role of the JAK-STAT pathway in myocardial injury. Trends Mol Med 2007; 13: $82-89$.

25. Cai ZP, Parajuli N, Zheng X, Becker L. Remote ischemic preconditioning confers late protection against myocardial ischemia-reperfusion injury in mice by upregulating interleukin-10. Basic Res Cardiol 2012; 107: 277.

26. Heusch G, Musiolik J, Kottenberg E, Peters J, Jakob H, Thielmann M. STAT5 activation and cardioprotection by remote ischemic preconditioning in humans: Short communication. Circ Res 2012; 110: $111-115$.

27. Slagsvold KH, Rognmo O, Høydal M, Wisløff U, Wahba A. Remote ischemic preconditioning preserves mitochondrial function and influences myocardial microRNA expression in atrial myocardium during 
coronary bypass surgery. Circ Res 2014; 114: 851-859.

28. Oba T, Yasukawa H, Hoshijima M, Sasaki KI, Futamata N, Fukui D, et al. Cardiac-specific deletion of SOCS-3 prevents development of left ventricular remodeling after acute myocardial infarction. $J \mathrm{Am}$ Coll Cardiol 2012; 59: 838-852.

29. Zhang W, Petrovic JM, Callaghan D, Jones A, Cui H, Howlett C, et al. Evidence that hypoxia-inducible factor-1 (HIF-1) mediates transcriptional activation of interleukin-1beta (IL-1beta) in astrocyte cultures. J Neuroimmunol 2006; 174: 63-73.

30. Matsuoka H, Nishida H, Nomura G, Van Vliet BN, Toshima H. Hypertension induced by nitric oxide synthesis inhibition is renal nerve dependent. Hypertension 1994; 23: 971-975.

31. Jelkmann W. Regulation of erythropoietin production. $J$ Physiol 2011; 589: $1251-1258$

32. Johns EJ, Kopp UC, DiBona GF. Neural control of renal function. Compr Physiol 2011; 1: 731-767.

33. Eckardt KU, LeHir M, Tan CC, Ratcliffe PJ, Kaissling B, Kurtz A Renal innervation plays no role in oxygen-dependent control of erythropoietin mRNA levels. Am J Physiol 1992; 263: F925-F930.

34. Fink GD, Fisher JW. Erythropoietin production after renal denervation or beta-adrenergic blockade. Am J Physiol 1976; 230: 508-513.

35. Ditting T, Hilgers KF, Stetter A, Linz P, Schönweiss C, Veelken R. Renal sympathetic nerves modulate erythropoietin plasma levels after transient hemorrhage in rats. Am J Physiol Renal Physiol 2007; 293: F1099-F1106.

36. Gebhard C, Petroktistis F, Zhang H, Kammerer D, Köhle C, Klingel $\mathrm{K}$, et al. Role of renal nerves and salt intake on erythropoietin secretion in rats following carbon monoxide exposure. J Pharmacol Exp Ther 2006; 319: 111-116.

37. Riksen NP, Hausenloy DJ, Yellon DM. Erythropoietin: Ready for prime-time cardioprotection. Trends Pharmacol Sci 2008; 29: 258 267.

38. Namiuchi S, Kagaya Y, Ohta J, Shiba N, Sugi M, Oikawa M, et al. High serum erythropoietin level is associated with smaller infarct size in patients with acute myocardial infarction who undergo successful primary percutaneous coronary intervention. J Am Coll Cardiol 2005; 45: 1406-1412.

39. Ferrario M, Massa M, Rosti V, Campanelli R, Ferlini M, Marinoni $\mathrm{B}$, et al. Early haemoglobin-independent increase of plasma erythropoietin levels in patients with acute myocardial infarction. Eur Heart J 2007; 28: 1805-1813.

40. Nakamura R, Takahashi A, Yamada T, Miyai N, Irie H, Kinoshita $\mathrm{N}$, et al. Erythropoietin in patients with acute coronary syndrome and its cardioprotective action after percutaneous coronary intervention. Circ J 2009; 73: 1920-1926.
41. Maiese K, Li F, Chong ZZ. New avenues of exploration for erythropoietin. JAMA 2005; 293: 90-95.

42. Hu J, Yan Y, Zhou Q, Ji M, Niu C, Hou Y, et al. Effects of renal denervation on the development of post-myocardial infarction heart failure and cardiac autonomic nervous system in rats. Int J Cardiol 2014; 172: e414-e416, doi:10.1016/j.ijcard.2013.12.254.

43. Hu J, Ji M, Niu C, Aini A, Zhou Q, Zhang L, et al. Effects of renal sympathetic denervation on post-myocardial infarction cardiac remodeling in rats. PLoS One 2012; 7: e45986, doi:10.1371/journal. pone.0045986.

44. Nozawa T, Igawa A, Fujii N, Kato B, Yoshida N, Asanoi H, et al. Effects of long-term renal sympathetic denervation on heart failure after myocardial infarction in rats. Heart Vessels 2002; 16: 51-56.

45. Loukogeorgakis SP, Panagiotidou AT, Broadhead MW, Donald A, Deanfield JE, MacAllister RJ. Remote ischemic preconditioning provides early and late protection against endothelial ischemia-reperfusion injury in humans: Role of the autonomic nervous system. $J$ Am Coll Cardiol 2005; 46: 450-456.

46. Liem DA, Verdouw PD, Ploeg H, Kazim S, Duncker DJ. Sites of action of adenosine in interorgan preconditioning of the heart. Am J Physiol Heart Circ Physiol 2002; 283: H29-H37.

47. Schoemaker RG, van Heijningen CL. Bradykinin mediates cardiac preconditioning at a distance. Am J Physiol Heart Circ Physiol 2000; 278: H1571-H1576.

\section{Supplementary Files}

Supplementary File 1

Figure S1. Effects of remote ischemic preconditioning (RIPC) and renal denervation (RND) on systemic blood pressure.

Figure S2. Laser Doppler image of renal blood flow (RBF) in mice with or without renal denervation (RND) at the indicated time after remote ischemic preconditioning (RIPC) ( $\mathrm{n}=7$ per group).

Figure S3. Serum levels of interleukin-6 (IL-6) and granulocyte colony-stimulating factor (G-CSF) were not altered after remote ischemic preconditioning (RIPC) in humans.

Figure S4. Before and immediately after RIPC, the kidneys were harvested and homogenized, and renal norepinephrine (NE) content was measured ( $n=9$ per group).

Please find supplementary file(s);

http://dx.doi.org/10.1253/circj.CJ-14-1171 